Received: 06/07/2018

Revision: 20/11/2018

Accepted: $25 / 11 / 2018$

OnlineFirst: 19/12/2018

\title{
The Role of Personal Best Goals in EFL Learners' Behavioural, Cognitive, and Emotional Engagement
}

\author{
Mostafa Hosseyni Ramshe \\ $\mathrm{PhD}$ candidate, Ferdowsi University of Mashhad, Iran, mostafa.h1367@yahoo.com
}

Mohammad Ghazanfari

Assoc. Prof., Ferdowsi University of Mashhad, Iran, mghazanfari@um.ac.ir

Behzad Ghonsooly

Prof., Ferdowsi University of Mashhad, Iran, ghonsooly@um.ac.ir

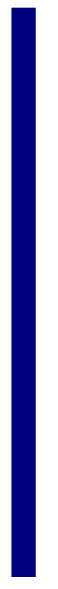

\begin{abstract}
There may be successful and less successful learners in every learning context. The latter group may try their best to improve their performance in comparison with their previous failures. To achieve such an objective, they try to adopt goals which are likely to promote their success. Such goals are commonly known as personal best goals. The purpose of the present study was to investigate the role of personal best goals in behavioural, cognitive, and emotional engagement of learners in an academic context. The participants of the study consisted of 302 B.A. university students majoring in English literature. One questionnaire consisting of items relating to different types of engagement and personal best goals was applied to collect the data of the study. Confirmatory factor analysis was conducted for each of the measures related to personal best goals, behavioural, emotional, and cognitive engagement, each showing an acceptable goodness of fit index. Moreover, a model was proposed. In the proposed structural equation model, personal best goals accounted for 55, 51, and 51 percent of variance in cognitive, behavioural, and emotional engagement, respectively.
\end{abstract}

Keywords: personal best goals, behavioural engagement, emotional engagement, cognitive engagement, structural equation modelling

\section{INTRODUCTION}

Goals are considered as an important source of motivation for learners to succeed, and perhaps what makes the learners different in terms of their success is the type of goals they set for their future. The kind of goals the learners adopt depends on whether they believe that they can change their current situation or not. In fact, successful and less successful learners seem to differ in how they view intelligence. Those with an

Citation: Ramshe, M. H., Ghazanfari, M., \& Ghonsooly, B. (2019). The Role of Personal Best Goals in EFL Learners' Behavioural, Cognitive, and Emotional Engagement. International Journal of Instruction, 12(1), 1627-1638. https://doi.org/10.29333/iji.2019.121103a 
incremental view toward intelligence believe that they can change their academic and non-academic outcomes through behavioural, emotional and cognitive modifications, whereas those with an entity view toward intelligence are less inclined to change their psycho-behavioural state (Dweck, 2012). Although some learners may not be able to perform as successfully as their own peers do, they can at least enhance their own performance in comparison with their own previous status.

Goals that are consistent with an incremental view of intelligence are labelled as personal best goals, defined as "personalized goals or standards of excellence that match or exceed one's previous best in an academic context'" (Martin \& Liem, 2010). They are challenging, specific, and competitively self-referenced objectives. Increasing one's efforts or performing better on current school activities than in the previous ones are examples of such goals.

Personal best goals emanated from growth approaches which were earlier referred to incremental view to human intelligence and value- added models. The term value-added has been borrowed from economics and refers to the amount of increase in the value of a product in each production stage (Koedel, Mihaly, \& Rockoff, 2015). In education, however, the term refers to how students' performance has improved in a specific educational year in comparison with the previous educational year and how teachers can help the students to improve their performance.

Research has shown that academic personal bests predict the degree of achievement and engagement in learners (for example, see Martin \& Liem, 2010). According to Martin (2012) personal best goals determine the desired target and help learners to perform better than what they have previously achieved. In addition, they help the learners pay attention to those activities essential for the accomplishment of the desired objectives. Moreover, as the kind of competition rising from the pursuit of such goals is with oneself, they bring the necessary energy for striving toward the favourable objectives. Additionally, personal best goals generate a kind of incongruence between your present situation and what you desire to achieve, motivating you to solve this incongruity. Finally, because personal best goals are naturally challenging, they put you under the necessary pressure for success.

In contrast with goals such as outperforming the other learners which are of a social and collectivist nature, personal best goals are personal and are of an individualistic nature. According to Hofstede, Hofstede, and Minkov (2010, p. 92):

Individualism pertains to societies in which the ties between individuals are loose. Everyone is expected to look after himself or herself and his or her immediate family. Collectivism, as its opposite, pertains to societies in which people from birth onward are integrated into strong, cohesive in-groups, which throughout people's lifetime continue to protect them in exchange for unquestioning loyalty.

As explained by Markus and Kitayama (1991), in collectivist settings, the "self" is defined in an interdependent fashion, where the individual is viewed as a tightly dependent member of the community. 
Thus, this creates a kind of contrast between the personal nature of personal best goals and the collectivist setting of some Asian countries like Iran (Hofstede et al., 2010).

Moreover, learning in an academic context is always accompanied with many achievements and failures. Those who experience the failure, try to do better than what they have done before. Thus, they try to resort to goals which lead to an improved performance and the adoption of personal best goals beside the other goals is one way to improve the past performances.

Therefore, this study intended to investigate the role of personal best goals in an Iranian academic context with the participation of the students majoring in English literature and examine whether such goals can help the learners to have a better experience of learning. Moreover, the researchers wish to discover whether there is any difference between boys and girls in terms of the adoption of such goals. Finally, as the Iranian society is considered to be a collectivist culture (Hofstede et al., 2010), the study is to investigate the role of personal best goals with a personal and individualistic nature in a collectivist setting.

\section{Review of the Literature}

Here, a brief history of the related concepts and studies is presented.

\section{Personal best goals}

Several theories like Achievement Goal Theory, Goal-setting Theory, Selfdetermination Theory, and Self-concordance Model are connected to growth approaches to human development.

Achievement Goal Theory (Kaplan \& Maehr, 2007) proposes that mastery and performance goals underlie achievement behaviours. Mastery goals are concerned with developing your competence and skills, showing your competence to others and trying to outperform them. It has been proposed that growth goals are a mixture of mastery and performance goals (Martin \& Liem, 2010). Such goals are mastery-oriented due to their self-referenced nature. They further consist of a performance component due to competing with one's own previous experiences.

Goal-setting Theory (Lock \& Latham, 2002) is another theory related to the growth approach. Based on this theory, growth goals help the learners to strive for those tasks and activities which are important for educational gains. As there is self-competition, they energize the learners and because of the lack of harmony between the current situation and the desired one, the learner is motivated to bridge this gap.

In addition, Self-determination Theory (Deci \& Ryan, 2008) proposes that three psychological needs including autonomy, relatedness, and competence must be met to lead to autonomous motivation which is an integrated and intrinsic form of motivation. Research suggests that autonomous motivation and growth goals operate in a similar fashion. The common point between growth goals and Self-determination Theory is that both of them are determined by learners and are selected on a self-based system. 
One more theory concerned with the self-referenced nature of growth goals is SelfConcordance Model (Sheldon \& Elliot, 1999). This model states that there should be a kind of harmony between goals and one's values and interests. Goals which are selfconcordant are integrated with one's self. In contrast, goals set externally are in contrast with one's interests and values and do not provide enough motivation.

Martin (2006) proposed a multidimensional model to investigate the main components of personal best goals in an educational setting. His model fits the data well and significantly predicted educational aspirations, persistence, enjoyment of school, and class participation. According to the proposed model, specific goals, challenging goals, self-referenced goals, and self-improvement goals formed the main cornerstones of personal best goals.

Personal best goals are specific outcomes, in the sense that there is less ambiguity in what must be achieved and the standards are clear. In addition, the level of challenge suggested by a personal best goal must be higher than what was previously considered as best level of performance. Situation-specific goals state the fact that personal best goals include obtaining a personalized standard which is compared with one's own previous level of performance. This includes competitive self-reference and selfimprovement; that is competition with one's own previous performance as well as extending previous levels of attainment or building upon previous achievements.

\section{Engagement}

According to $\mathrm{Hu}$ and Kuh (2002), engagement is defined as "the quality of effort students themselves devote to educationally purposeful activities that contribute directly to desired outcomes". Fredricks, Blumenfeld, and Paris (2004) define three dimensions for engagement: behavioural, emotional, and cognitive. Behavioural engagement is concerned with the norms of behaviour like involvement and attendance and the avoidance of negative or disruptive behaviour. Interest, enjoyment and a sense of belonging are affective reactions experienced by those being emotionally engaged. Finally, learners who are cognitively engaged invest in their own learning, look for more than what is expected of them and enjoy challenge. Each of these different forms of engagement has a positive and negative pole. This means that meeting the expectations shows internalization and approval and failure to meet the norms can be disruptive. For example, a student with a positive mode of cognitive engagement meets or exceeds assignment requirements, whereas a student with a negative mode of cognitive engagement gives a new definition of the parameters for assignments and the late submission of the assigned homework is an example of non-engagement.

\section{Studies on personal best goals}

Martin, Collie, Mok, and McInerney (2016) examined personal goal structure, individual personal best goals, engagement, and achievement in Chinese and English speaking background students in Australian schools. They found that individual personal best goals were related to engagement and engagement was associated with achievement. In addition, Yu and Martin (2014) examined the role of personal best goals and classic achievement goals (mastery and performance goals) in predicting 
academic motivation, engagement and buoyancy in a Chinese context. Their results showed that mastery and personal best goals predicted variance in motivation, engagement, and academic buoyancy outcomes.

Moreover, Liem, Ginns, Martin, Stone, and Herrett. (2012) investigated the role of personal best goals in academic and social functioning. Longitudinal structural equation modelling showed that at time 1, PB goals significantly predicted students' deep learning, academic flow, positive teacher relationships, academic buoyancy, and favourable attitudes toward peer cooperation. At time 2, the effects of personal best goals on academic flow, deep learning, and positive teacher relationship was still significant after the prior variance of corresponding time-1 factors were controlled. In addition, Mok, Wong, Su, Tognolini, and Stanley investigated whether personal best goals and self-regulation could predict mathematics achievement through a multilevel structural equation model in Hong Kong. The results showed that students' personal best goals affected their achievement. In contrast self-regulation indirectly affected mathematics achievement through personal best goals and it did not directly affect mathematics achievement. In another study conducted by Collie, Martin, Bapworth, and Ginns (2016) the association of interpersonal relationships with parents, teachers and peers with personal best goals and academic engagement in US, Canada, and UK were examined. The results indicated that students' perceptions of teachers, parents, and peers were associated with personal best goals and academic engagement, while teacher and peer relationship indirectly affected cognitive and behavioural engagement through personal best goals.

The study of personal best goals seems to be still in its infancy and the number of the studies conducted in this area of research does not appear to be adequate (to the best of our knowledge, so far, about ten studies have been conducted in this area). Thus, it is felt that more studies are needed to enrich our understanding of such goals and their role in different academic contexts around the world, with learners of various cultural and educational backgrounds. Thus, this study tries to contribute its share to expand our existing knowledge about the issue in question in a collectivist Iranian culture where there have been no adequate studies and see how personal best goals operate in the context of the present study regarding their personal nature which is in contrast to the collectivist nature of the Iranian society.

\section{METHOD}

\section{Participants}

Convenience sampling procedure was employed to select the participants of the study and availability was considered as the criterion for the selection of the participants. As the data of the present study is part of a Ph.D. thesis conducted in the field of teaching English as a foreign language, BA English literature students with upper-intermediate and advanced levels of language proficiency were chosen as the participants. The respondents included 302 students (88 males, 206 females, and 8 of unspecified gender) studying in two state universities in Mashhad and Isfahan as well as two private universities in Mashhad. Their average age ranged from 18 to 22 years old. 


\section{Instruments}

Personal best goals scale

Four items from Martin and Liem's study (2010) were taken to measure personal best goals (e.g., when I do school work, I try to do it better than I've done before).

\section{Behavioural engagement scale}

Five items of ongoing engagement subscale of Rochester Assessment Package for Schools (RAPS) (Wellborn \& Connell, 1987) were taken to measure behavioural engagement.

\section{Emotional engagement scale}

Four items of Affect to School subscale of Facilitating Conditions for School Motivation (McInerney, Dowson, \& Yeung, 2005) constituting the emotional engagement were chosen to measure this construct.

\section{Cognitive engagement scale}

This scale consisted of three subscales of cognitive engagement mentioned in Goal Orientation and Learning Strategies Survey (Goal-S) (Dowson \& McInerney, 2004). It consists of six items for organization (e.g., I try to organize my school notes when I want to learn things for school), six items for Rehearsal (e.g., when I want to learn things for school I reread my notes), and six items for Elaboration (e.g., I try to understand how the things I learn in school fit together with each other).

\section{Data Collection Procedure and Data Analysis}

The items related to personal best goals, behavioural engagement, cognitive engagement, and emotional engagement were all combined to form a single questionnaire. The questionnaire was presented in English as the medium of instruction in all the classes was English. It was distributed by the first author among the students in one session after obtaining students' and teachers' permission.

It should be noted that in order to resolve any misunderstanding concerning the questionnaire items, it had been already administered in a pilot study. 30 students participated in the pilot study and the reliability score (the Cronbach Alpha) for the scale was 0.916 . As the questionnaire items have been previously used and modified in other studies, there was no misunderstanding with the items and the student fully understood what the items mean. Moreover, the students selected their answers to questionnaire items from a Likert scale consisting of five choices and each choice being assigned a number for further statistical analysis (strongly agree $=5$, agree $=4$, neither agree nor disagree $=3$, disagree $=2$, strongly disagree $=1$ ). 


\section{FINDINGS}

\section{Correlation among the variables, the reliability and the mean of the scales}

The mean score for the personal best goals was 3.85 with the Cronbach Alpha being 0.87. All the scales showed a good Cronbach Alpha score, except for the Behavioural Engagement Scale, which is marginally acceptable. In addition, personal best goals were significantly and positively correlated with all the three facets of engagement.

Table 1

Correlation among the Variables

\begin{tabular}{|c|c|c|c|c|c|c|}
\hline & $\begin{array}{l}\text { Personal } \\
\text { Best Goals }\end{array}$ & $\begin{array}{l}\text { Behavioural } \\
\text { Engagement }\end{array}$ & $\begin{array}{l}\text { Emotional } \\
\text { Engagement }\end{array}$ & Elaboration & Monitoring & Regulating \\
\hline Personal Best Goals & & $.350^{* * *}$ & $.368^{* *}$ & $.376^{* *}$ & $.417^{* * *}$ & $.300^{* * *}$ \\
\hline Behavioural Engagement & & & $.389^{* *}$ & $.284^{* * *}$ & $.398^{* * *}$ & $.341^{* * *}$ \\
\hline Emotional Engagement & & & & $.401^{* *}$ & $.407^{* *}$ & $.306^{* *}$ \\
\hline Elaboration & & & & & $.525^{* *}$ & $.309^{* * *}$ \\
\hline Monitoring & & & & & & $.532^{* *}$ \\
\hline Mean & 3.858 & 3.360 & 3.833 & 4.008 & 3.882 & 3.748 \\
\hline Std. Deviation & 0.774 & 0.629 & 0.700 & 0.604 & 0.607 & 0.602 \\
\hline Cronbach's Alpha & 0.879 & 0.595 & 0.631 & 0.887 & 0.838 & 0.728 \\
\hline
\end{tabular}

\section{Gender effects on personal best goals}

The results of the ANOVA statistic showed that males and females differed significantly in terms of their support for personal best goals $(\mathrm{F}=9.478$, Partial eta 0.031) and females' support (3.944) for these goals was higher than the male (3.855) counterparts. Table 2 provides the results related to ANOVA and descriptive statistics related to gender.

Table 2

ANOVA results for gender.

\begin{tabular}{lllllll}
\hline & Gender & $\mathrm{N}$ & Mean & Std. Deviation & $\mathrm{F}$ & Partial eta \\
\hline Personal & male & 88 & 3.6477 & .90706 & & \\
Best & female & 206 & 3.9442 & .68203 & \multirow{2}{*}{$9.478 * *$} & 0.031 \\
nyyyls & Total & 294 & 3.8554 & .76697 & & \\
\hline
\end{tabular}

\section{The validity of the instrument}

To determine the construct validity of the scales, confirmatory factor analysis (CFA) was applied. Four CFAs were conducted for personal best goals, cognitive engagement, behavioral engagement, and emotional engagement. All the scales showed good fit indices. Table 3 provides goodness of fit statistics. 
Table 3

Goodness of fit statistics

\begin{tabular}{lllllll}
\hline \multicolumn{1}{c}{ Model } & CMIN/DF & RMSEA & \multicolumn{1}{c}{ GFI } & IFI & TLI & CFI \\
\hline Personal best goals & 0.962 & 0 & 0.998 & 1 & 1 & 1 \\
\hline Cognitive engagement & 2.765 & 0.077 & 0.882 & 0.906 & 0.887 & 0.905 \\
\hline Behavioural engagement & 1.904 & 0.055 & 0.99 & 0.986 & 0.964 & 0.986 \\
\hline Emotional engagemen & 1.985 & 0.057 & 0.994 & 0.989 & 0.966 & 0.989 \\
\hline Desired Level & $1-5$ & $>0.8$ & $>0.9$ & $>0.9$ & $>0.9$ & $>0.9$
\end{tabular}

Note: $R M S E A=$ root mean square error of approximation, GFI=goodness of fit index, $I F I=$ incremental fit index, TLI=Tucker-Lewis index, CFI=comparative fit index, CMIN/DF=normalized chi-square/degrees of freedom

\section{Structural equation modelling}

Based on the assumption that Personal Best Goals affect each of the different forms of engagement, namely, behavioural engagement, emotional engagement, and cognitive engagement, a model was developed. It should be noted that the developed model was postulated based on the previous literature showing an effect for personal best goals on various aspects of engagement. In order to have a better model, one item form the behavioural engagement scale accounting for just .03 percent of variance in this scale was deleted.

In this model, there are some lines drawn from the circles showing the amount of error for each item which indicate the amount of error which is common among these items. The model enjoys an acceptable goodness of fit statistic $(\chi 2=1.827, \mathrm{df}=80, \mathrm{P}$ value $=.000$, $\mathrm{RMSEA}=.052$, $\mathrm{IFI}=.960, \mathrm{TLI}=946, \mathrm{CFI}=.959$ ). Thus, the model is confirmed. Moreover, personal best goals affected cognitive, behavioural, and emotional engagement with significant coefficients of 55, 51, and 51 percent, respectively. 


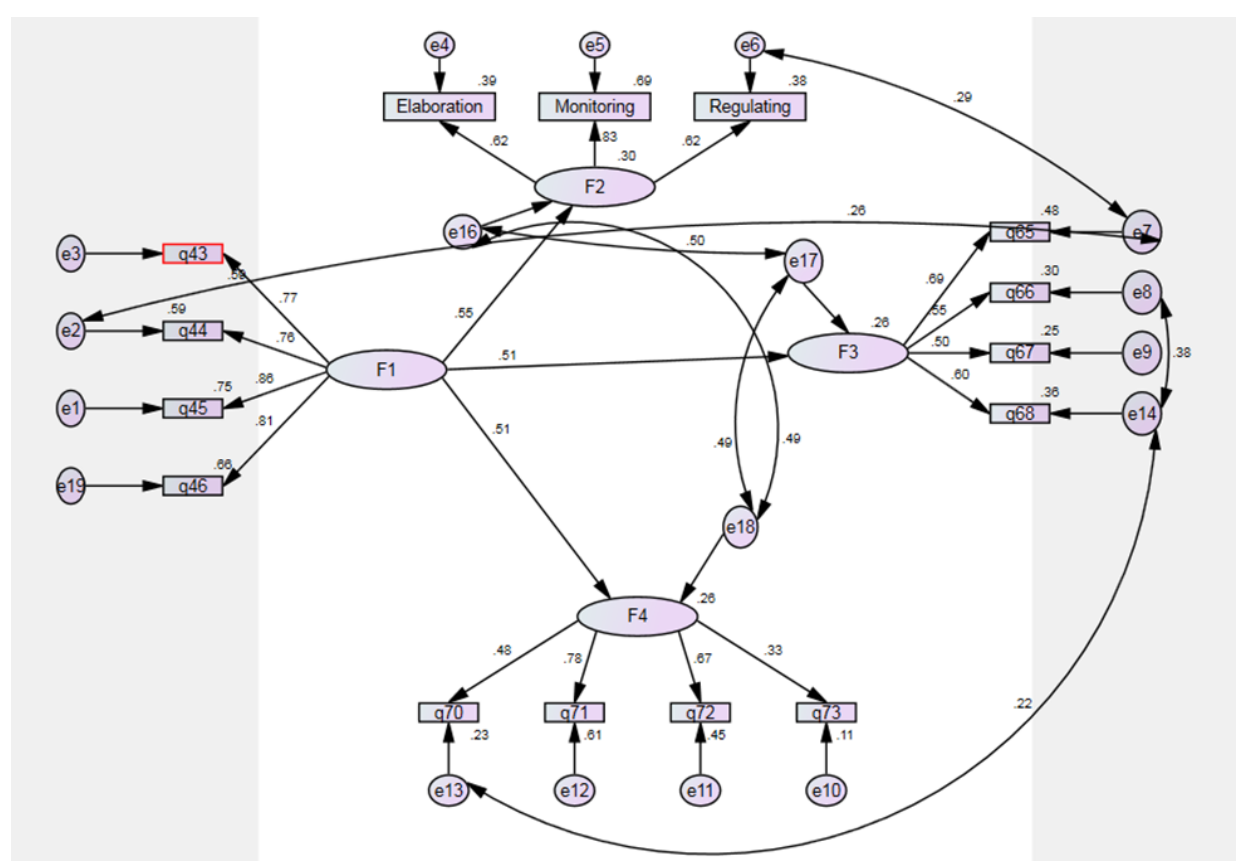

Note: F1: personal best goals, F2: cognitive engagement, F3: behavioural engagement, F4: emotional engagement

Figure 1

The Structural Equation Model for the Effect of Personal Best Goals on Different Facets of Engagement

\section{DISCUSSION}

Personal best goals were highly endorsed by the students and they accounted for a significant amount of variance in all the aspects of engagement. They are somehow free from a specific context and their adaptive role has been confirmed in different contexts like Hong Kong (Mok, Wong, Su,Tognolini, \& Stanely, 2014) and Australia (Martin \& Elliot, 2015). Although the Iranian society is considered as a collectivist society (Hofstede et al., 2010) and these goals as the name suggests are personal and not concerned with social issues, this high degree of support for personal best goals indicates their applicability in the Iranian culture. The results also corroborate the findings of Martin et al. (2016) in China, a collectivist society, who found an association between personal best goals and engagement. Similarly, the study by Martin (2014) showed that personal best goals accounted for a distinct amount of variance in academic engagement and buoyancy.

As noted by Segall, Lonner, and Berry (1998), the aim of cross-cultural psychology is to test our current knowledge of psychology in other cultures than where they were originated, to discover the local characteristics of the phenomenon, and to build a universal theory being applicable to all cultures. This study in fact tested the 
applicability of a notion created by Western psychologists in an Eastern culture and the applicability of the personal best goals was confirmed in the Iranian culture.

Moreover, Martin and Liem (2010) proposed that self-regulation strategies and personal best goals are of the same nature due to their emphasis on the concepts of "self" and "personal"; therefore self-regulated learners may include among their goals personal best ones as well. Pertinent to their claim, in this study, engagement was defined in terms of self-regulation and self- monitoring, two forms of metacognitive engagement, and personal best goals predicted a significant amount of variance in both of these two forms of engagement. As Yu and Martin (2014) have indicated personal best goals are connected with engagement and this is because personal best goals are more observable and action-oriented (put more effort on the current task).

As it has been noted by Martin (2006), individuals with a personal goal orientation take their own previous performance as the standard for deciding whether they have been successful with regard to the task at hand and this causes a lower amount of pressure on the students due to the goal of absolute attainment of the task inherent in mastery goals or making a comparison between their own performance and others' which is the characteristic of performance goals. It is this kind of improvement which is capable of paving the way for the students' future successes or overcoming their current failures, even when there is little improvement from the other people's perspective. With regard to the beneficial role attributed to the personal best goals in this study, teachers in academic contexts like the one discussed in this study, should prioritize self-referenced comparisons over other-referenced ones to make the learning environment more motivating for the learners.

The personally-indexed nature of growth inherent in personal best goals is in fact what is called Personal Best Growth Percentile (PBGP) Models of assessment (Martin, 2011). In such models, there is a baseline for the growth of each student and assessment is made on the basis of students' previous growth; each student's growth being determined across time without any reference to the performance of others. Thus, this model of assessment can be applied as a tool to help the students improve their own performance without the stress accompanied by the comparison made between their performance and the others' performance.

In addition, males and females differed significantly in terms of their support for personal best goals with female students' support for such goals being higher than the male ones. This is in line with the findings by Martin (2006), who found that the adoption of personal best orientation is more common among the females than the males. Moreover, as it was noted in another study by Martin (2004), there has been a declining trend in males' motivation and due to the relevance of personal best goals to achievement and motivation; this can be regarded as a justification for the fact that girls are more inclined towards such goals in comparison to boys.

\section{CONCLUSION}

Any learner is supposed to set various kinds of goals for his or her learning, goals which are likely to affect the quality of learning to a great extent. In fact, these are the goals 
which guide the person in the complex road of learning. One major type of the goals that are expected to pave the way for one's learning are personal best goals. The adoption of this type of goals has proven to lead to clear positive outcomes due to their obvious insistence on improvement. This study investigated the role of personal best goals in different facets of engagement. Our findings suggest that that they are effective for learners' behavioural, emotional, and cognitive engagement. Thus, in any learning situation, learners should be reminded of their potential positive effects. While corroborating the findings of the studies conducted in individualistic Western cultures, this study provides evidence for the claim that such goals seem to be beneficial for the learners in the collectivist Iranian culture as well.

\section{REFERENCES}

Collie, R. J., Martin, A. J., Bapworth, B., \& Ginns, P. (2016). Students' Interpersonal Relationships, Personal Best (PB) Goals, and Academic Engagement. Learning and Individual Differences, 45, 65-76.

Deci, E. L., \& Ryan, R. M. (2008). Facilitating Optimal Motivation and Psychological Well-being across Life's Domains. Canadian Psychology, 49 (1), 14-23.

Dowson, M., \& McInerney, D. M. (2004). The Development and Validation of the Goal Orientation and Learning Strategies Survey (GOALS-S). Educational and Psychological Measurement, 64 (2), 290-310.

Dweck, C. S. (2012). Mindsets and Human Nature: Prompting Change in the Middle East, the Schoolyard, the Racial Divine, and Willpower. American Psychologist, 67 (8), 614-622.

Fredricks, J. A., Blumenfeld, P. C., \& Paris, A. H. (2004). School Engagement: Potential of the Concept, State of the Evidence. Review of Educational Research, 74 (1), 59-109.

Hofstede, G., Hofstede, G. J., \& Minkov, M. (2010). Cultures and organizations: software of the mind: intercultural cooperation and its importance for survival. (3rd ed.). New York; London: McGraw-Hill.

Hu, S., \& Kuh, G. D. (2002). Being (dis) engaged in Educationally Purposeful Activities: The Influence of Student and Institutional Characteristics. Research in Higher Education, 43 (5), 555-575.

Kaplan, A., \& Maehr, M. L. (2007). The Contributions and Prospects of Goal , Orientation Theory. Educational Psychology Review, 192, 141-184.

Koedel, C., Mihaly, K., \& Rockoff, J. E. (2015). Value-added Modeling: A Review, Economics of Education Review, 4(C), 180-195.

Locke, E. A., \& Latham, G. P. (2002). Building a Practically Useful Theory of Goal Setting and Task Motivation. American Psychologist, 57(9), 705-717.

Markus, H. R., \& Kitayama, S. (1991). Culture and the self: implications for cognition, emotion, and motivation. Psychological Review, 98(2), 224-253. 
Martin, A. J. (2004). School Motivation of Boys and Girls: Differences of Degree, Differences of Kind or Both?. Australian Journal of Psychology, 56(3), 133-146.

Martin, A. J. (2006). Personal Bests (PBs): A Proposed Multidimensional Model and Empirical Analysis. British Journal of Educational Psychology, 7(4), 803-825.

Martin, A. J. (2011). Personal Best (PB) Approaches to Academic Development: Implications for Motivation and Assessment. Educational Practice and Theory, 33(1), 93-99.

Martin, A. J. (2012). The Role of Personal Best (PB) Goals in the Achievement and Behavioral Engagement of Students with ADHD and Students without ADHD. Contemporary Educational Psychology, 37(2), 91-105.

Martin, A. J., \& Elliot, A. J. (2015). The Role of Personal Best (PB) and Dichotomous Achievement Goals in Students' Academic Motivation and Engagement: A Longitudinal Investigation. Educational Psychology, 34 (5), 1285-1302.

Martin, A. J., \& Liem, G. A. D. (2010). Academic Personal Bests (PBs), Engagement, and Achievement: A Cross-Lagged Panel Analysis. Learning and Individual Differences, 20(3), 265-270.

Martin, A. J., Collie, R. J., Mok, M. M.C., \& McInerney, D. M. (2016). Personal Best (PB) Goal Structure, Individual PB Goals, Engagement, and Achievement: A Study of Chinese- and English-Speaking Background Students in Australian Schools. British Journal of Educational Psychology, 86(1), 75-91.

McInerney, D. M., Dowson, M., \& Yeung, A. S. (2005). Facilitating Conditions for School Motivation. Educational and Psychological Measurement, 65(6), 1046-1066.

Mok, M. M C., Wong, M. Y W., Su, M. R.,Tognolini, J., \& Stanely, G. (2014). Personal Best Goal and Self-Regulation as Predictors of Mathematics Achievement: A multilevel Structural Equation Model. Asia Pacific Journal of Educational Development, 3(1), 7991.

Segall, M. H., Lonner, W. J., \& Berry, J. W. (1998). Cross-Cultural Psychology as a Scholarly Discipline. American Psychologist, 53 (10), 1101-1110.

Sheldon, K. M., \& Elliot, A. J. (1999). Goal Striving, Need Satisfaction, and Longitudinal Well-Being: The Self-Concordance Model. Journal of Personality and Social Psychology, 76 (3), 482-497.

Yu, K., \& Martin, A. J. (2014). Personal Best (PB) and 'Classic' Achievement Goals in the Chinese Context: Their Role in Predicting Academic Motivation, Engagement, and Buoyancy. Educational Psychology, 34(5), 635-658.

Wellborn, J. G., \& Connell, J. P. (1987). Student engagement and disaffection in school student report. Rochester, NY: Rochester Assessment Package for Schools. 